\title{
Pediatric Liver Transplantation: A Surgical Perspective and New Concepts
}

\author{
Sukru Emre • Gabriel E. Gondolesi • A. Salim Muñoz-Abraham • \\ Gulus Emre • Manuel I. Rodriguez-Davalos
}

Published online: 11 October 2014

(C) Springer International Publishing AG 2014

\begin{abstract}
The first successful liver transplantation was carried out in 1967, and the recipient was a pediatric patient. Since then, many challenges have been overcome and, nowadays, 1-year patient survival after liver transplantation is about $90 \%$ in pediatric patient populations. Standardization of surgical technique, development of better immunosuppressive medications, improved understanding of caring for patients with end-stage liver disease, and improvements in anesthesia and intensive care have had a tremendous impact on graft and patient survival. Despite all improvements, challenging issues in pediatric liver transplantation remain. In this article, we address some of these problems.
\end{abstract}

Keywords Pediatric liver transplantation - Segmental liver transplantation · Split liver transplantation · Early postoperative complications $\cdot$ Hepatic artery thrombosis . PELD

\section{Introduction}

Liver transplantation (LT) is the gold standard of treatment for end-stage liver disease and some metabolic/genetic liver diseases in pediatrics, with an excellent 1-year survival rate reaching above $90 \%$. These exceptional results have been achieved through the standardization of surgical procedures; surgical innovations such as split liver transplantation (SLT)

S. Emre $(\bowtie) \cdot$ A. S. Muñoz-Abraham • G. Emre •

M. I. Rodriguez-Davalos

Department of Surgery, Division of Transplantation and

Immunology, Yale University School of Medicine, 330 Cedar Street,

FMB 121, New Haven, CT 06510, USA

e-mail: sukru.emre@yale.edu

G. E. Gondolesi

Fundacion Favaloro-University Hospital, Buenos Aires, Argentina and living donor liver transplantation (LDLT); improvement of intensive care treatment; development of new immunosuppressive medications; improved understanding of the balance between immunosuppression and opportunistic infection; and the adoption of a multidisciplinary team approach to patient care, which includes collaboration between transplant surgery, pediatric hepatology, pediatric intensive care, nephrology, pulmonary, cardiology, infectious disease, psychiatry, social work, pharmacy, and nutrition.

Despite these good outcomes, many challenges remain in pediatric LT. Appropriate patient selection, organ shortage and the organ allocation algorithm, time of transplantation, technical problems, and opportunistic infections are some of the problems faced by transplant programs on a daily basis. This chapter addresses some of these issues.

\section{Indications and Contraindications for Liver Transplantation}

Indications for LT are depicted in Table 1. Approximately 40$50 \%$ of the children who require LT have biliary atresia (BA); the next most common indications are pediatric acute liver failure (PALF), metabolic/genetic liver diseases, other cholestatic liver diseases, autoimmune hepatitis, and malignant tumors. Absolute contraindications for LT include severe brain injury and mitochondrial disease, among others (Table 1). Even when a patient does not have an absolute contraindication for LT, there are some circumstances in which the decision to move forward to transplant must be weighed against other medical co-morbidities. One such situation would be the decision to transplant a child with the possibility of developing brain herniation in the setting of PALF. Although the transplant team may be willing to perform a transplant despite the risk of losing the patient secondary to herniation, the decision should always be made based on 
Table 1 Indication and contraindications in pediatric liver transplantation

\begin{tabular}{|c|c|}
\hline \multicolumn{2}{|l|}{ Indications } \\
\hline $\begin{array}{l}\text { Cholestatic liver } \\
\text { disease }\end{array}$ & $\begin{array}{l}\text { - Biliary atresia } \\
\text { - Progressive familial intrahepatic cholestasis } \\
\text { - Syndromic bile duct paucity (Alagille } \\
\text { syndrome) } \\
\text { - Non-syndromic bile duct paucity } \\
\text { - Primary sclerosing cholangitis (PSC) } \\
\text { - Neonatal hepatitis } \\
\text { - Choledochal cyst } \\
\text { - Total parenteral nutrition (TPN)-associated } \\
\text { cholestasis }\end{array}$ \\
\hline $\begin{array}{l}\text { Metabolic liver } \\
\text { disease }\end{array}$ & $\begin{array}{l}\text { - Wilson's disease } \\
\text { - } \alpha 1 \text { - antitrypsin deficiency } \\
\text { - Tyrosinemia type } 1 \\
\text { - Glycogen storage diseases } \\
\text { - Crigler-Najjar syndrome } \\
\text { - Maple syrup urine disease } \\
\text { - Familial hypercholesterolemia } \\
\text { - Arginase deficiency } \\
\text { - Propionic acidemia } \\
\text { - Urea cycle defects } \\
\text { - Neonatal hemochromatosis }\end{array}$ \\
\hline Liver tumors & $\begin{array}{l}\text { - Hepatoblastoma } \\
\text { - Hepatocellular carcinoma } \\
\text { - Hemangioendothelioma } \\
\text { - Neuroendocrine Tumors } \\
\text { - Nested Stromal Epithelial Tumors }\end{array}$ \\
\hline Acute liver failure & $\begin{array}{l}\text { - Idiopathic } \\
\text { - Drug-induced } \\
\text { - Autoimmune hepatitis } \\
\text { - Infectious } \\
\text { - Toxins }\end{array}$ \\
\hline Miscellaneous & $\begin{array}{l}\text { - Cystic fibrosis } \\
\text { - Vascular malformations } \\
\text { - Congenital hepatic fibrosis } \\
\text { - Chronic hepatitis } \\
\text { - Chronic hepatitis C }\end{array}$ \\
\hline \multicolumn{2}{|l|}{ Contraindications } \\
\hline $\begin{array}{l}\text { Absolute } \\
\text { contraindications }\end{array}$ & $\begin{array}{l}\text { - Coma with irreversible brain injury } \\
\text { - Uncontrolled systemic sepsis } \\
\text { - Terminal progressive systemic disease } \\
\text { - Severe cardiopulmonary disease } \\
\text { - Generalized mitochondrial disease }\end{array}$ \\
\hline $\begin{array}{l}\text { Relative } \\
\text { contraindications }\end{array}$ & $\begin{array}{l}\text { - Extrahepatic metastasis disease } \\
\text { - Pulmonary hypertension } \\
\text { - Psychosocial and adherence problems in } \\
\text { teenagers }\end{array}$ \\
\hline
\end{tabular}

objective criteria, such as thorough neurological examination, imaging study results, electroencephalography (EEG), and intracranial pressure monitoring. Since brain herniation can occur upon reperfusion of the allograft, anesthesia care, including fluid management during the hepatectomy and reperfusion, is of paramount importance. Another situation would be the decision to transplant a child who presents with PALF due to mitochondrial disease. Since mitochondrial diseases are systemic problems, LT may not change the dismal outcome for the patient. To further complicate the decision-making process, a final diagnosis of mitochondrial disease may take several weeks to confirm, and often LT may need to be performed sooner than that in order to save the life of the child. When mitochondrial disease is suspected, it is advisable to discuss the possibility of systemic disease, related problems, and outcomes with parents.

\section{Early Referral, Pre-Transplant Work-Up, and Management}

Early referral to a transplant center is important and allows for state-of-the-art evaluation, management, and preparation of the patient for LT. Additionally, a transplant center is well equipped to educate the patient and the family as well as to address medical issues, such as managing the complications of end-stage liver disease and nutritional support. Patients referred to a transplant center will be evaluated by a multidisciplinary team, including transplant surgery, hepatology, transplant coordinators, social workers, nutritionists, pharmacists, psychiatrists, cardiologists, anesthesiologists, and, if necessary, by other disciplines based on the patient's disease and other co-morbidities. As part of the patient assessment, we also perform a complete laboratory workup, including but not limited to a comprehensive metabolic panel, complete blood count $(\mathrm{CBC})$, prothrombin time (PT)/partial thromboplastin time (PTT)/international normalized ratio (INR), serologies (cytomegalovirus [CMV], Epstein-Barr virus [EBV], hepatitis $\mathrm{C}$ virus [HCV], hepatitis B virus [HBV], human immunodeficiency virus [HIV]), autoimmune markers, and a hypercoagulable workup. Imaging studies are also important to delineate liver vascular and biliary anatomy as well as to diagnose any potential underlying malignancies. The preferred imaging modality is magnetic resonance imaging (MRI) and Doppler ultrasound; we prefer not to use computed tomography (CT) scans with our pediatric patients so as to minimize radiation exposure.

\section{Timing of Liver Transplantation}

The decision of when to perform LT is a dynamic balancing act. While performing early LT in a compensated cirrhotic individual can lead to unnecessary morbidity and mortality, performing a late transplant could also decrease the chance of survival after LT secondary to progression of the illness and decompensation of the patient. Therefore, performing LT in a timely fashion is a key factor in achieving successful outcomes. In general, moving forward to transplant is indicated when the patient develops serious complications of liver cirrhosis such as esophageal variceal bleeding, ascites, failure to thrive, worsening synthetic functions, jaundice, and pruritus. 
Decisions about timing of LT can also be considered according to diagnostic indication for LT. For example, the timing of transplant in patients with BA, the most common diagnosis in pediatric LT, is often discussed. In children who have undergone a Kasai procedure (portoenterostomy), LT is not indicated as long as their serum bilirubin is normal and there is no growth failure or signs of ascites and cholangitis. It is our policy that LT is necessary when the patient has experienced more than three cholangitis episodes requiring hospitalization and intravenous antibiotic treatment within a 1-year period. Furthermore, LT is also necessary when the Kasai procedure fails with no drainage of bile. In these cases, we prefer not to wait for the development of the serious complications of end-stage liver disease before performing LT.

The timing of LT for metabolic/genetic liver diseases is also challenging. In these cases, close collaboration with the pediatric genetic specialist, pediatric hepatology, and transplantation is essential. In urea cycle defect (UCD) cases, it is essential to perform LT before patients develop a severe metabolic crisis that could result in permanent neurological damage. We can also perform hepatocyte transplantation as a bridge to LT 1-3 months after birth while the patient waits for a suitable donor organ; this procedure allows the patient to grow to a more suitable size and age for LT without developing a metabolic crisis. Tyrosinemia is one particular metabolic disease in which children can develop hepatocellular carcinoma in their native liver as early as 3 months of age (personal experience). Therefore, these patients should be monitored closely, and LT needs to be offered in a timely fashion.

\section{Organ Allocation, Donor Selection, and Donor/Recipient Matching}

The current organ allocation algorithm in the USA is the Pediatric End-Stage Liver Disease (PELD) scoring system for children aged less than or equal to 12 years. However, many publications have shown that the PELD system is not an accurate reflection of the degree of illness for most of the children waiting for LT $[1,2]$. Furthermore, disparities in the current organ allocation system in the USA have been proven [3••]. Despite flaws in the pediatric organ allocation system, pediatric waiting list mortality is lower than that of the adult waiting list due to the greater success rate of performing LDLT or SLT in children. Furthermore, a United Network for Organ Sharing (UNOS) organ allocation policy indicates that pediatric deceased donor livers should be allocated to pediatric recipients, which increases the chances of a child on the waiting list receiving a size-matched, whole-organ transplant [4]. Needless to say, the experience and capability of the pediatric transplant team in performing state-of-the-art procedures such as LDLT and SLT is very important for decreasing waiting list mortality. For example, at the Yale University
Transplant Center, the waiting list mortality for pediatric patients is $<2 \%$ because our surgeons are well trained to perform innovative segmental LT techniques.

Recipient body size and donor liver size matching is a key factor for success in pediatric LT, especially in children aged less than 1 year. Although small-for-size syndrome is a major obstacle in performing LDLT in adults, in pediatrics this is not an issue. Conversely, in pediatric LDLT, large-for-size is a problem. LDLT can be carried out successfully with a graft/ recipient weight ratio (GRWR) of up to 4. In cases of GRWR $>4$, primary closure of the abdomen would be impossible and may result in infection or vascular problems [5]. Due to the small size of pediatric patients (especially those under 3 months old), it is sometimes preferable to wait until the patient grows so that they will be more likely to find a suitable size allograft. Although it is technically feasible to perform a successful LT in children even less than 1 month of age, many times it is impossible to find an allograft of an acceptable size, even when the splitting technique is utilized. Overall, it is advisable to balance the risk of early transplant with a larger sized donor allograft with the serious complications of endstage liver disease and the related potential neurocognitive developmental delays [6].

\section{Acute Liver Failure}

Up to $13 \%$ of LTs conducted in children are performed in the setting of PALF; interestingly, ALF is the indication for transplant in only $7 \%$ of adults $[7,8]$. ALF is defined as the sudden loss of hepatic function in a patient with no pre-existing liver disease. The presence of elevated liver enzymes and the development of a coagulopathy (INR $>1.5$ ) are essential for the diagnosis of PALF with or without the development of encephalopathy in PALF. Approximately $17 \%$ of the children who needed a transplant or died from PALF never developed hepatic encephalopathy (HE) [9]. This may be because HE is difficult to recognize in children and that it may only manifest in final stages of the disease.

PALF has a varied presentation. A recent publication by Squires and the PALF Study Group described PALF as heterogeneous and dynamic and postulated that it could be due to a novel or unrecognized virus with or without exposure/ toxicity related to acetaminophen. While a great number of cases have an indeterminate etiology, autoimmune processes, infection, and metabolic/drug toxicity causes have been identified as causal for PALF $[8,10]$. The majority of PALF cases present within 2 weeks, but sub-acute presentations have also been documented. Under the current allocation system in the USA, children with a confirmed diagnosis of ALF are listed with the highest priority as UNOS Status 1-A [4]. Despite assigning the highest priority to the children with PALF, due to the national organ shortage, transplant surgeons sometimes 
still have to rely on LDLT or auxiliary partial orthotopic liver transplantation (APOLT) for these patients.

Our team has previously published the use of LDLT as an option for patients with PALF [11]. In our study, the average waiting time was statistically shorter in the LDLT group (4 days) than in children who received a deceased donor LT (7.25 days). In our series, both the LDLT and the deceased donor transplant groups had $100 \% 1$-year survival. It is our policy to use LDLT as a back-up option in the setting of PALF. When the patient's condition deteriorates and there is no suitable deceased donor available, LDLT becomes an absolutely life-saving procedure. Recently, a group from South Korea described their experience with 47 children receiving LDLT and reported a 1-year survival rate of $81.9 \%$ [12].

If there is a chance of native liver regeneration and recovery in the future, APOLT would be an excellent option. APOLT preserves the part of the diseased organ that has the potential to regenerate after the acute injury and transplants the portion of the segmental graft. The end goal of this procedure is to eventually stop immunosuppression once the native liver has been able to regenerate adequate function [13].

$\mathrm{ABO}$ incompatible organs may be utilized as a last resort when there is no blood type-matched organ available. ABOincompatible organ usage can only be justified in an emergency situation. Centers with experience in $\mathrm{ABO}$ incompatibility routinely use a combination of plasmapheresis and intravenous immunoglobulin (IVIG) followed by a dose of rituximab to prevent rejection [14]. In children under the age of 2 years, these strategies may not be necessary, but close follow-up of the appropriate antibody titers is imperative [15].

\section{Pre-Transplant Nutrition}

It is well known that there is a direct relation between liver disease and malnutrition; in fact, one of the important components of the PELD score system is the degree of growth failure of the patient in question [16]. Growth issues and failure to thrive are very common reasons for transplant centers to ask for 'exemption points' to move a patient up on the list. As was mentioned previously, the timing of transplant is also dependent on the physical and neuro-developmental state of the candidate, both of which are correlated with nutritional status.

Every patient who initiates a transplant work-up must be assessed by a certified registered dietitian. An assessment of global nutrition prior to LT helps guide team management of nutrition peri- and post-operatively, which in turn promotes recovery and helps to prevent infection. In fact, one study showed that the use of enteral nutrition within $48 \mathrm{~h}$ of transplant in LDLT recipients actually reduces the incidence of bacterial sepsis significantly. Every patient should also get anthropomorphic measurements as part of the evaluation. In our program, the dietician works in collaboration with the pediatric hepatologist to educate parents in the pre-transplant phase and to make decisions about nutrition (for example, whether or not tube feeding is needed) to optimize weight prior to transplant, especially for patients with BA.

Before LT, patients can be hyper- or hypometabolic, and their diet should be individualized. Concentrated formulas with high medium-chain triglycerides (MCTs) are encouraged but must be adjusted individually, especially in cases of certain metabolic liver diseases [17]. Since our patients are usually receiving calcineurin inhibitors (CNIs) and corticosteroids after LT, which both contribute to insulin resistance, we stress the importance of tight glycemic control post-LT to avoid the complications of hyperglycemia. Many of our patients who are dependent on enteral nutrition prior to LT, especially those with cholestatic liver disease like BA, may need to continue the enteral feeds after LT until they meet their nutritional goals [17].

\section{Current Technical Options in Pediatric Liver Transplant}

Despite the progressive gap that exists between patients in need and organ availability, the field of pediatric LT is one of the few specialties in which the evolution of surgical technique has allowed transplant programs to almost abolish mortality on the waiting list. This has been achieved with the use of cadaveric whole liver transplants, and other innovative techniques, including reduced size LT (RLT), SLT, and LDLT. In the following section, we describe the common technical aspects of LT, discuss the differences between techniques, and the potential pitfalls of each type of graft.

\section{Common Technical Hurdles in Pediatric Liver Transplantation}

Pediatric LT usually begins with bilateral subcostal incisions, which are adequate to obtain enough visualization in patients younger than 5 years. Beyond this age, or whenever is necessary, midline extension of the incision is indicated in order to obtain adequate exposure. The next step, performing the hepatectomy, can be simple or can be technically challenging if the patient has undergone a previous Kasai procedure or other upper abdominal surgeries. A history of such surgeries may lead to the formation of dense adhesions. Together with the development of portal hypertension secondary to liver cirrhosis, these adhesions make the hepatectomy phase extremely difficult. This part of the procedure also has the highest rate of bleeding and requires careful dissection to avoid injuries to the duodenum, colon, or adjacent vascular structures. The use of electrocautery during hepatectomy may cause thermal injury to abdominal organs, specifically the small and large intestine. 
It is difficult to recognize these injuries during the operative period because perforation-related symptoms usually occur between the 3rd and 5th post-operative day.

Although one may assume a poorer outcome in patients with a history of the Kasai procedure, due to the technical challenges, our results as well as those of others have shown that prior Kasai procedure actually does not influence the survival of patients, nor does it increase the incidence of perioperative complications [18-20].

\section{Liver Transplant Variants}

\section{Whole Liver Transplantation}

We perform all of our LT operations with the piggyback technique. The supra hepatic vena cava anastomosis is the key factor for establishing good venous return to the heart. The surgeon must pay careful attention to this anastomosis, making sure to avoid kinking, telescoping, or narrowing of the anastomosis, since these complications could cause out-flow obstruction. Surgeons should be prepared to use the triangulation technique (if necessary), and age-appropriate suture material needs to be used.

In the construction of the portal vein (PV) anastomosis, it is crucial to avoid both redundancy and tension. In cases of size discrepancy between donor and recipient PVs, equal distribution of size mismatch along the course of the anastomosis is of paramount importance to ensure excellent laminar flow, thereby avoiding PV thrombosis (PVT). In cases of BA, it is important to recognize the existence of an atretic PV. Once the atretic $\mathrm{PV}$ is recognized, extending the PV dissection to the level of the spleno-mesenteric junction is indicated. The PV anastomosis can be done at the level of the spleno-mesenteric junction by using a vascular graft after the removal of the atretic segment. In small children, we perform PV anastomoses with a $7 / 0$ prolene running suture for the posterior wall and interrupted sutures for the anterior wall. Since we use non-absorbable suture material, this technique allows for the growth of the vessel as the patient grows without causing PV stricture.

Surgeons must strive for perfect construction of the hepatic artery (HA) anastomosis since HA thrombosis is the leading cause of graft failure [21]. We prefer the use of interrupted 8/0 prolene sutures for HA anastomoses in vessels with a diameter $<5 \mathrm{~mm}$. Using microsurgical instruments with fine surgical technique and avoiding kinking, twisting, and tension is essential for excellent outcomes. We do not think using a surgical microscope is necessary; we have achieved $100 \% \mathrm{HA}$ patency using surgical loupes with 3.5 or 4.5 magnification.

Biliary reconstruction can be done either as a duct-to-duct (DD) anastomosis or a Roux-Y-hepatico-jejunostomy [22]. In BA cases with a history of failed Kasai procedure, the previously created Roux loop can be used if it has adequate length and quality. If possible, a DD reconstruction is recommended. If recipient bile duct size does not allow for the performance of any of the above, a cholecysto-jejunostomy could be used.

\section{Segmental Liver Transplantation}

The anatomical studies performed by Couinaud in 1954 established the basis for future technical developments in liver surgery [23]. He described the anatomical concept of segmental liver anatomy, establishing that "the liver was constituted by eight segments having independent arterial, venous and biliary pedicles and unique liver drainage." Segments II and III constitute the left lateral liver segment (LLS), which is the most commonly used segmental graft in pediatric LT. Use of a left lobe (LL) (Seg II-III and IV) graft is preferred for larger children or adolescents.

\section{Reduced Size Liver Transplantation}

RLT is the first described surgical innovation in the field of LT. The development of RLT has allowed surgeons to evolve their understanding of liver anatomy and led to the development of SLT and LDLT [24]. This procedure reduced pediatric waiting list mortality by using an adult liver for a pediatric recipient, but unfortunately the unused portion of the viable liver is discarded. Currently, RLT is only used when a whole deceased donor liver is not suitable for splitting between an adult and pediatric patient or one side of the liver is not usable secondary to traffic accident-related damage.

\section{Split Liver Transplantation}

The concept of splitting the liver to be used in two different recipients has increased liver transplant applicability. At the end of the procedure, two complete liver grafts can be obtained, most commonly an LLS for a pediatric recipient and the extended right lobe (segment IV-VIII plus I) for a teenager or an adult. Although right (segment V to VIII) and left (segment I to IV) splitting can be done, it did not gain popularity secondary to technical challenges and graft size problems resulting in an increased incidence of primary graft non-function, vascular thrombosis, bile leaks, or sepsis [25]. SLT can be carried out either on the back table (ex vivo splitting) or during the procurement (in situ splitting). The in situ technique is preferable since it minimizes the post-perfusion bleeding from the cut surface of the liver, and more importantly shortens the cold ischemia time for both liver allografts [26].

Previous experience with SLT has also provided the knowledge to establish standards and selective donor criteria for splitting. These changes have made the current results of SLT comparable to those of whole liver transplants or LDLT, encouraging different countries around the globe to establish policies that utilize the splitting technique [27]. 
Table 2 Vascular complications and management options

\begin{tabular}{lll}
\hline Occurrence of complication & Early & Late \\
\hline $\begin{array}{l}\text { Management options } \\
\text { Hepatic artery thrombosis }\end{array}$ & Thrombectomy or retransplantation & $\begin{array}{c}\text { No intervention for hepatic artery thrombosis, management of biliary } \\
\text { complications ( }+/- \text { Retransplantation if clinically indicated) } \\
\text { Interventional radiology, balloon dilatation }\end{array}$ \\
$\begin{array}{l}\text { Hepatic artery stenosis } \\
\text { Portal vein thrombosis }\end{array}$ & $\begin{array}{l}\text { Anticoagulation and close follow-up } \\
\text { Consider revising the anastomosis }\end{array}$ & $\begin{array}{c}\text { Interventional radiology balloon dilation }+/- \text { stent placement. If IR } \\
\text { unsuccesful Meso-Rex bypass or selective porto-systemic shunt } \\
\text { Meso-Rex bypass or selective porto-systemic shunt }\end{array}$ \\
\hline
\end{tabular}

\section{Living Donor Liver Transplant}

LDLT consists of a healthy volunteer giving a portion, approximately $25-60 \%$ of his or her own liver to a patient in need. The evolution of the procedure and the ethical considerations related to the small but real risk of death for a healthy person has limited its use in occidental countries. Therefore, in the USA, LDLT is performed with donor safety as the number one priority; furthermore, centers that perform LDLT are regulated by UNOS and the Center for Medicaid and Medicare Services (CMS). Pediatric recipients usually need a smaller portion of the liver; therefore, risk to the donor is lower in pediatric LDLT. Advantages of performing LDLT include better availability of liver allograft when LT is necessary, shorter ischemia time, and better allograft quality. In general, the donors must be healthy volunteers between 18 and 55 years of age, with normal liver function, compatible blood type, and no other health issues. An assigned team who is not involved in the care of the recipient evaluates the donor. The team includes an independent donor advocate team, a gastroenterologist, a transplant surgeon, and a cardiologist. Laboratory work up includes but is not limited to a hypercoagulable state workup; a relevant serological workup; and an evaluation of donor vasculature, biliary structure, and whole liver volume/graft segment volume via CT/MRI [28-30].

The living donor surgery for a pediatric recipient can be performed using open surgery or a minimally invasive approach. The surgical details of both techniques have been explained elsewhere [28, 31-33]. Performing the donor hepatectomy laparoscopically reduces post-operative morbidity and shortens recovery time for the donor [34].

Principles of the recipient surgery for LDLT are explained at the beginning of this section.

\section{Post-Operative Care}

Early post-operative care of an LT patient is truly a team effort. We prefer to send our patients to the pediatric intensive care unit (PICU) while they are still intubated and sedated immediately after we complete their surgery. Following pertinent liver function tests and vitals, managing ventilator settings and fluid-electrolyte levels, as well as monitoring urinary output are all part of standard post-operative care. Routine lab tests include liver function tests (LFTs) (alanine aminotransferase [ALT], aspartate aminotransferase [AST], bilirubin, alkaline phosphatase, gamma-glutamyl transpeptidase [GGTP]), serum lactate levels, serum albumin, serum fibrinogen, $\mathrm{PT} /$ PTT/INR, basic metabolic panel, and CBC. These labs are all drawn every 6 hours during the first post-operative day. The diagnosis of post-operative vascular complications can be made with adequate screening techniques. In our group, we routinely perform a Doppler ultrasound at the end of the procedure to get an idea of baseline vascular functioning, which we compare with Dopplers performed on postoperative days 1, 2, and 3 [11]. We also perform an annual ultrasound, as recommended by the SPLIT (Studies of Pediatric Liver Transplantation) Research Committee.

We recommend early extubation as soon as is safe after transplantation. We believe that early extubation decreases pulmonary infectious complications and overall length of hospital stay.

Postoperative Surgical Complications - Vascular and Biliary

Biliary complications are the Achilles heel of LT and include biliary leakage (from anastomoses or cut surface of the liver) and biliary strictures. Biliary leaks will occur as an early postoperative complication and increases morbidity and mortality after LT [35]. Biliary leaks may occur secondary to technical errors or HA thrombosis. Treatment of bile leaks can be handled either surgically or by interventional radiology. Bile duct strictures usually occur as a late post-operative complication and do not generally impact morbidity and mortality post-operatively. Nowadays, most bile duct strictures are managed by interventional radiology, and surgical correction is rarely needed. Early diagnosis and timely treatment of bile duct strictures is necessary to avoid progression of the stricture to the extent that it cannot be corrected by interventional radiology.

Although vascular complications are not as common as biliary or infectious complications, they have a much higher impact on patient and graft survival $[36,37]$. In order to review this complex topic, complications should be classified as being arterial or venous and as occurring early or late in the 
post-operative course, since these factors can determine treatment and predict outcome (Table 2).

In general, the incidence of vascular complications in pediatric LT varies between 2 and $30 \%$. Vascular complications are the major reason for graft loss in LDLT [36, 37]. A study from the University of California, Los Angeles (UCLA) group found that segmental grafts had a significantly higher incidence of vascular complications ( $4 \%$ in SLT, $8.7 \%$ in LDLT, $1.7 \%$ in whole) [37]. In our experience, vascular complications can be minimized regardless of graft type, although we believe that grafts from very small donors $(<10 \mathrm{kgs})$ may have a higher incidence of vascular complications due to their size and vessel characteristics. Imaging studies such as CT/MR angiography are non-invasive and are the preferable diagnostic tools for the discovery of vascular complications.

The treatment of HA or PV complications depends on when the problem is diagnosed. If HA thrombosis (HAT) does occur, it can present in one of three ways: as a bile leak, with elevated liver enzymes, or without any clinical/lab changes. If HAT occurs with no clinical/lab change, the chance of salvaging the graft via thrombectomy is high. Patients who present with enzyme elevation or bile duct necrosis leading to biliary leakage have a lower chance of successfully salvaging the live allograft by thrombectomy. Therefore, these patients can be re-listed as UNOS status $1 \mathrm{~A}$, but only if they are diagnosed with HAT within 2 weeks of the initial surgery [4]. Otherwise, they must be re-listed according to their PELD/MELD (Model for End-Stage Liver Disease) score at the time of HAT diagnosis.

HA stenosis (HAS) is an issue that is being diagnosed more often with the current advances in imaging. In our recently published series, we found HAS occurring at an incidence of $2.8 \%$ [36]. The cases may often present with biliary issues, and we recommend treatment with balloon angioplasty without stenting.

In the case of PVT, if there is early detection of thrombosis, revision with thrombectomy is required. For the management of late extra-hepatic PVT that presents with portal hypertension, we believe the Meso-Rex bypass should be performed when feasible. A recent study compared the use of percutaneous transluminal angioplasty (PTA) vs. Meso-Rex bypass for PV stenosis or PVT in growing pediatric recipients. This study showed that the Meso-Rex bypass, which redirects the portal flow to the liver, can be a superior alternative when compared with using PTA with stenting [38].

\section{Conclusion}

LT is an excellent treatment modality, with exceptional 1-, 5-, and 10-year graft and patient survival rates in children with end-stage liver disease, ALF, and metabolic disorders. While surgical skill is obviously important for the completion of a successful transplant surgery, recovery and long-term survival is made possible through the utilization of a protocol-driven, team-based approach to caring for the patient. Despite progress that has been made, the future still holds many challenges. Some of the first successful pediatric liver transplant recipients have now had their grafts for over 3 decades, and we are just beginning to understand long-term problems and their impact on transplanted liver. For example, liver fibrosis has been seen to develop for no apparent reason and without a change in liver functions in patients who have had their grafts for more than 5 years. Antibody-mediated rejection, chronic side effects of long-term immunosuppression, recurrent disease, tolerance induction, and transition into adulthood will be the other hurdles to overcome in order to increase longevity of transplanted livers and further improve the quality of life after LT.

\section{Compliance with Ethics Guidelines}

Conflict of Interest Sukru Emre, Gabriel E. Gondolesi, A. Salim Muñoz-Abraham, Gulus Emre, and Manuel I. Rodriguez-Davalos declare that they have no conflict of interest.

Human and Animal Rights and Informed Consent This article does not contain any studies with human or animal subjects performed by any of the authors.

\section{References}

Papers of particular interest, published recently, have been highlighted as:

•. Of major importance

1. Shneider BL, Neimark E, Frankenberg T, Arnott L, Suchy FJ, Emre S. Critical analysis of the pediatric end-stage liver disease scoring system: a single center experience. Liver Transpl. 2005;11(7):78895 .

2. Shneider BL, Suchy FJ, Emre S. National and regional analysis of exceptions to the pediatric end-stage liver disease scoring system (2003-2004). Liver Transpl. 2006;12(1):40-5.

3.• Gentry SE, Chow EK, Wickliffe CE, Massie AB, Leighton T, Segev DL. Impact of broader sharing on transport time for deceased donor livers. Liver Transplant. 2014. This paper explains the complexity of the organ allocation system and its consequences in the USA.

4. Organ Procurement and Transplantation Network. Policies. Richmond (VA); 2014.

5. Kasahara M, Sakamoto S, Umeshita K, Uemoto S. Effect of graft size matching on pediatric living-donor liver transplantation in Japan. Exp Clin Transplant. 2014;12 Suppl 1:1-4.

6. Rosencrantz R, Cimsit B, Seashore M, Arvelakis A, Kulkarni S, Ghiroli $\mathrm{M}$, et al. Pediatric liver transplantation for inherited 
metabolic liver disease: a single-center experience. Transplant Proc. 2011;43(3):896-900.

7. Sundaram V, Shneider BL, Dhawan A, Ng VL, Im K, Belle S, et al. King's college hospital criteria for non-acetaminophen induced acute liver failure in an international cohort of children. J Pediatr. 2013;162(2):319.e1-23.e1.

8. Squires Jr RH, Shneider BL, Bucuvalas J, Alonso E, Sokol RJ, Narkewicz MR, et al. Acute liver failure in children: the first 348 patients in the pediatric acute liver failure study group. J Pediatr. 2006;148(5):652.e2-8.e2.

9. Sundaram SS, Alonso EM, Narkewicz MR, Zhang S, Squires RH. Characterization and outcomes of young infants with acute liver failure. J Pediatr. 2011;159(5):813.e1-8.e1.

10. Lee WM, Seremba E. Etiologies of acute liver failure. Curr Opin Crit Care. 2008;14(2):198-201

11. Emre S, Rodriguez-Davalos M, Umman V, Cartiera K, Wollwerth $\mathrm{S}$, Arnott L, et al. Liver transplantation at Yale-new haven transplantation center. Clin Transpl. 2011;187-201.

12. Oh SH, Kim KM, Kim DY, Kim Y, Song SM, Lee YJ, et al. Improved outcomes in liver transplantation in children with acute liver failure. J Pediatr Gastroenterol Nutr. 2014;58(1): 68-73.

13. Belghiti J, Sommacale D, Dondero F, Zinzindohoue F, Sauvanet A, Durand F. Auxiliary liver transplantation for acute liver failure. HPB (Oxford). 2004;6(2):83-7.

14. Wu J, Ye S, Xu X, Xie H, Zhou L, Zheng S. Recipient outcomes after ABO-incompatible liver transplantation: a systematic review and meta-analysis. PLoS One. 2011;6(1):e16521.

15. Gelas T, McKiernan PJ, Kelly DA, Mayer DA, Mirza DF, Sharif K. $\mathrm{ABO}$-incompatible pediatric liver transplantation in very small recipients: Birmingham's experience. Pediatr Transplant. 2011;15(7):706-11.

16. Freeman RB, Wiesner RH, Harper A, McDiarmid SV, Lake J, Edwards E, et al. The new liver allocation system: moving toward evidence-based transplantation policy. Liver Transpl. 2002;8(9): $851-8$.

17. Ikegami T, Shirabe K, Yoshiya S, Yoshizumi T, Ninomiya M, Uchiyama $\mathrm{H}$, et al. Bacterial sepsis after living donor liver transplantation: the impact of early enteral nutrition. J Am Coll Surg. 2012;214(3):288-95.

18. Altman RP, Lilly JR, Greenfeld J, Weinberg A, van Leeuwen K, Flanigan L. A multivariable risk factor analysis of the portoenterostomy (kasai) procedure for biliary atresia: twenty-five years of experience from two centers. Ann Surg. 1997;226(3):34853. discussion 353-5.

19. Cowles RA, Lobritto SJ, Ventura KA, Harren PA, Gelbard R, Emond JC, et al. Timing of liver transplantation in biliary atresia-results in 71 children managed by a multidisciplinary team. $\mathrm{J}$ Pediatr Surg. 2008;43(9):1605-9.

20. Visser BC, Suh I, Hirose S, Rosenthal P, Lee H, Roberts JP, et al. The influence of portoenterostomy on transplantation for biliary atresia. Liver Transpl. 2004;10(10):1279-86.

21. Olthoff KM, Merion RM, Ghobrial RM, Abecassis MM, Fair JH, Fisher RA, et al. Outcomes of 385 adult-to-adult living donor liver transplant recipients: a report from the A2ALL consortium. Ann Surg. 2005;242(3):314-23. discussion 323-5.

22. Starzl TE, Esquivel C, Gordon R, Todo S. Pediatric liver transplantation. Transplant Proc. 1987;19(4):3230-5.

23. Couinaud C. Lobes et segments hepatiques: notes sur l'architecture anatomique et chirurgicale du foie. Press Med. 1954;62.

24. Broelsch CE, Emond JC, Thistlethwaite JR, Rouch DA, Whitington PF, Lichtor JL. Liver transplantation with reducedsize donor organs. Transplantation. 1988;45(3):519-23.

25. Emre S, Umman V, Cimsit B, Rosencrantz R. Current concepts in pediatric liver transplantation. Mt Sinai J Med. 2012;79(2):199213

26. Rogiers X, Malago M, Habib N, Knoefel WT, Pothmann W, Burdelski M, et al. In situ splitting of the liver in the heart-beating cadaveric organ donor for transplantation in two recipients. Transplantation. 1995;59(8):1081-3.

27. Emre S, Umman V. Split liver transplantation: An overview. Transplantation proceedings. Elsevier; 2011.

28. Miller CM, Gondolesi GE, Florman S, Matsumoto C, Munoz L, Yoshizumi T, et al. One hundred nine living donor liver transplants in adults and children: a single-center experience. Ann Surg. 2001;234(3):301,11.

29. Emre S. Living-donor liver transplantation in children. Pediatr Transplant. 2002;6(1):43-6.

30. Shneider BL, Emre S. Pediatric liver transplantation: past, present, and future. Liver Transpl. 2006;12(4):511-3.

31. Broelsch CE, Burdelski M, Rogiers X, Gundlach M, Knoefel WT, Langwieler $\mathrm{T}$, et al. Living donor for liver transplantation. Hepatology. 1994;20(S7):S49-55.

32. Rogiers X, Burdelski M, Broelsch C. Liver transplantation from living donors. Br J Surg. 1994;81(9):1251-3.

33. Marcos A, Fisher RA, Ham JM, Shiffman ML, Sanyal AJ, Luketic VA, et al. Right lobe living donor liver transplantation. Transplantation. 1999;68(6):798-803.

34. Cherqui D, Soubrane O, Husson E, et al. Laparoscopic living donor hepatectomy for liver transplantation in children. Lancet. 2002;359(9304):392-6.

35. Varotti G, Gondolesi GE, Goldman J, Wayne M, Florman SS, Schwartz ME, et al. Anatomic variations in right liver living donors. J Am Coll Surg. 2004;198(4):577-82.

36. Rodriguez-Davalos MI, Arvelakis A, Umman V, Tanjavur V, Yoo PS, Kulkarni S, et al. Segmental grafts in adult and pediatric liver transplantation: improving outcomes by minimizing vascular complications. JAMA Surg. 2014;149(1):6370.

37. Duffy JP, Hong JC, Farmer DG, Ghobrial RM, Yersiz H, Hiatt JR, et al. Vascular complications of orthotopic liver transplantation: experience in more than 4,200 patients. J Am Coll Surg. 2009;208(5):896-903.

38. Cho Y, Kim K, Ha T, Ko G, Hwang J, Park H, et al. Management of late-onset portal vein complications in pediatric living-donor liver transplantation. Pediatr Transplant. 2014;18(1):64-71. 